\title{
Spacing mechanisms within light-induced copepod swarms
}

\author{
Andrew W. Leising ${ }^{1, *}$, Jeannette Yen $^{2}$ \\ 'Scripps Institution of Oceanography, University of California, San Diego, La Jolla, California 92093-0227, USA \\ ${ }^{2}$ Marine Science Research Center, State University of New York, Stony Brook, New York 11794-5000, USA
}

\begin{abstract}
Many species of copepods form dense aggregations, known as swarms. In the laboratory, we experimentally induced 5 different species of copepod to swarm in response to a point source of light. To map out the $(x, y, z, t)$ positions of swarm members, 2 right-angle views of the 3-dimensional swarm were videotaped. Since images of individual copepods appear indistinguishable on the paired 2-dimensional projections, an algorithm was developed which matched the temporal changes of the vertical $(z)$ positions of all images from the 2-dimensional projections of the 3-dimensional copepod movement to produce $(x, y, z, t)$ positions of each individual. With the temporal/spatial positional data of swarm members, we tested the hypothesis that the fluid disturbance surrounding individual moving copepods, rather than the exoskeleton, maintains minimum separation distance. As the density of the swarm increased, the average nearest-neighbor distance NND decreased, as did the mean minimum NND (MNND). For 3 of the 5 speries, the MNND was significantly greater than that predicted from a random distribution, and was greater than twice the antennule or prosome length. While occasional physical contact may occur, resulting in escapes or attempted matung, it appears that most swarm members remain outside the field of self-generated fluid motion in the boundary layers surrounding their neighbors.
\end{abstract}

KEY WORDS: Nearest neighbor distance Copepod swarms Matching methods Mechanoreception Escape response Antenna Hydrodynamic Mating behavior

\section{INTRODUCTION}

The overwhelming majority of planktonic species show over-dispersed or aggregated dispersal patterns (Haury \& Wiebe 1982, Omori \& Hamner 1982), with significant non-random variations in horizontal position (Wiebe 1970). Patchiness of organisms makes it difficult to sample them quantitatively and to assess natural population sizes (Omori \& Hamner 1982), which hampers our ability to quantify trophic cycling of energy. Determining the spatial distribution of aggregated populations is critical to understanding how a species maintains itself, as average food concentrations are often too low to support the observed growth of individuals within the population were these concentrations perfectly dispersed (Davis et al. 1991).

Copepods are often found in very dense aggregations known as swarms. Swarms can be defined as

•E-mall: aleising@ucsd.edu dense patches in which the individuals are not aligned parallel to one another, in contrast to schools where the individuals are all aligned parallel to one another and are swimming in a common direction (Clutter 1969). Swarming behavior may result from an attempt to increase mating encounters, food exploitation, and predator avoidance (as reviewed by Yen \& Bryant 1992) or to maintain position within the environment (Clutter 1969, Hamner \& Carlton 1979).

In the natural environment, one physical factor that may induce a swarm is the occurrence of sharp spatial gradients in light intensity. Examples of this type of swarm induction have been observed above patches of pale substrate that reflect more light than the surrounding dark substrata (Hebert 1980), above coral reefs which reflect light more than the surrounding water (Hamner \& Carlton 1979), and within the beams of light formed beneath and between the roots of mangrove trees (Ambler et al. 1991). In temperate waters, Acartia spp. has also been observed swarming above 
light colored objects on the bottom of small shallow bays (Udea et al. 1983). The concentration of copepods within swarms may reach several orders of magnitude higher than non-swarming densities (Omori \& Hamner 1982)

Photic stimuli draw copepods together locally as an aggregate, while individually there must be some mechanism which keeps them apart. Understanding the mechanisms which maintain spacing between individuals in a swarm should allow calculation of maximum swarm densities. Schooling fish use visual cues along with lateral-line sensory information to keep in formation and maintain their distance from one another (Partridge \& Pitcher 1980). Most copepods lack eyes capable of providing image cues, but do have an extremely acute mechanosensory system, the setae on their first antennae (Strickler \& Bal 1973, Gill 1986, Yen et al. 1992). These antennae extend laterally from the head, increasing the extent of the copepod's perceptive field. This may allow physical detection of local disturbances in the water made by other zooplankton (Haury et al. 1980, Lenz \& Yen 1993). Copepods also have sensory hairs located along their body, primarily on their prosome, augmenting those on the first antennae (Strickler 1975).

Copepods are capable of perceiving objects larger than themselves coming towards them before physical contact using remote mechanoreception (Haury et al. 1980). In response to either direct stimulation of the antennal mechanoreceptors (Gill 1985) or the close approach of a large object (Haury et al. 1980), an escape reaction is elicited, characterized by a shortduration, high-speed burst of swimming Copepods also can detect objects smaller than themselves at a distance, such as food particles entrained in the feeding current, although there is debate as to whether this is purely through mechanoreception, or mechanoreception plus chemoreception (Paffenhöfer et al. 1982. Legier-Visser et al. 1986, Paffenhöfer \& Lewis 1990). It is likely that mechanoreception is important in the perception of conspecifics and provides a means by which copepods could avoid all contact with one another while swarming (Strickler 1975). Strickler (1975) raises the important point, however, that at certain times this avoidance reaction must be suppressed so that mating may occur

Swarms often can be monospecific and of a single sex, reaching abundances of 1.5 million $\mathrm{m}^{-3}$ (Hamner \& Carl.ton 1979). Zooplankters swimming within such a swarm do not attack or mate with each other. They appear to remain together as a group aggregation yet individually, they separate in space. The separation distance appears to include the physical body of the plankters as well as a shell of water. From studies of the feeding current surrounding a moving copepod
(Strickler 1982, Yen et al. 1991, Fields \& Yen 1993) we saw that the copepod can create a fluid disturbance that is greater than the volume of the exoskeleton. Other studies also showed that mechanoreceptive setae are able to detect the flow velocities found in the feeding current (Yen et al. 1992).

Here we propose that the copepod and its fluid disturbance should be considered as a unit where not only does the physical organism occupy a space but so does its accompanying boundary layer of water. It is this volume that can be detected by neighboring copepods and that separates closely packed members of a zooplankton swarm. We tested the null hypothesis that the minimum mean nearest-neighbor distance (MNND. defined as the closest that 2 copepods will come to one another) between randomly distributed copepods within a swarm would decrease as density increased. The alternative to this hypothesis was that the MNND would be independent of density, indicating that the copepods were maintaining a fixed volume around themselves free from others and were not randomly distributed in space. An estimate of the minimum separation distance enables calculation of the density of the swarm and can reveal information about the perceptive range of a copepod. Several different species of copepod were separately induced to swarm in a small tank while being videotaped at high magnification. We used light to induce swarm formation, and developed computer software to solve the 'matching' problem (Ikawa et al. 1994), and to calculate the 3-dimensional (3D) coordinates of all individuals of a swarm over time. Of the 5 species studied, 3 showed an ability to maintain a minimum inter-individual distance, which was found to be proportional to the mean antenna length of each species.

\section{METHODS}

Five species of copepod were used for this experiment. Coullana canadensis nauplii were used from a laboratory culture that originated in Maine, USA. Adult Acartia tonsa, Oithona sp., and a benthic harpacticoid were collected using a $102 \mu \mathrm{m}$ mesh net from the inlet to Stony Brook harbor on Long Island Sound, NY, USA. Diaptomus sp. were collected from Lake Ontario near Rochester, NY, also using a $102 \mu \mathrm{m}$ mesh net. Copepods first were gathered from the sample buckets using a fiber-optic light to concentrate them at 1 spot, and then transferred to smaller beakers. Not all of the copepods in the bucket came towards the light. Thus, only individuals showing positive phototaxis were included in the experiment. Copepods were sorted by species under a dissecting microscope and placed into beakers of filtered sea water. An anesthetizing agent, 
Table 1 Density in test tank (in ind. $\mathrm{ml}^{-1}$, number of copepods in tank given in parentheses), and swarm density (in ind. $\mathrm{ml} \mathrm{l}^{-1}$, number of copepods per swarm given in parentheses) for each time sequence

\begin{tabular}{|c|c|c|c|c|c|c|}
\hline Species & Tank & Swarm 1 & Swarm 2 & Swarm 3 & Swarm 4 & Swarm 5 \\
\hline Acartia tonsa & $1.08(81)$ & $12.39(9)$ & $22.04(8)$ & $10.09(13)$ & $6.66(13)$ & $11.83(17)$ \\
\hline Harpacticoid & $0.97(73)$ & $16.9(16)$ & $19(18)$ & $20(21)$ & $39.8(10)$ & $42(22)$ \\
\hline Diaptomus sp. & $1.37(103)$ & $19.2(16)$ & $19(17)$ & $15.7(22)$ & - & - \\
\hline Oithona sp. & $1.57(118)$ & $6.4(8)$ & $8.2(9)$ & $14.6(16)$ & $11.2(22)$ & $19.2(16)$ \\
\hline Coullana canadensis & $0.5(50)$ & $224(10)$ & $291(13)$ & $353(18)$ & $294(15)$ & $269(12)$ \\
\hline
\end{tabular}

$m$-aminobenzoic acid ethyl ester methanesulfonate (MS-222, CalBiochem), was used to facilitate the capture and separation of copepods with minimal damage to their antennae and little effect on their behavior (Yen 1985). Each of the 5 species studied was collected at the time when it constituted the major if not the sole species type in the water column, facilitating the separation process. Within several minutes of being returned to fresh-filtered sea water after anesthetization, the copepods exhibited normal swimming behavior and appeared undamaged under the microscope.

The procedure for the 5 experimental trials, 1 trial for each species, was as follows: once separated by species, 50 to 118 copepods, depending on the species (Table 1), were transferred to the experimental test tank, a $100 \mathrm{ml}$ cubical glass container holding approximately $75 \mathrm{ml}$ of filtered sea water (or fresh for Diaptomus sp.). The copepods were allowed to acclimate to the test tank in total darkness at room temperature for at least 1 h before the experiment. Two chargedcoupled device (CCD) video cameras, mounted at right angles to each other, were used at high magnification, with optical paths similar to those developed by Strickler (1985) (Fig. 1). The copepods were videotaped for 10 min at 30 frames $\mathrm{s}^{-1}$ from both cameras simultane-

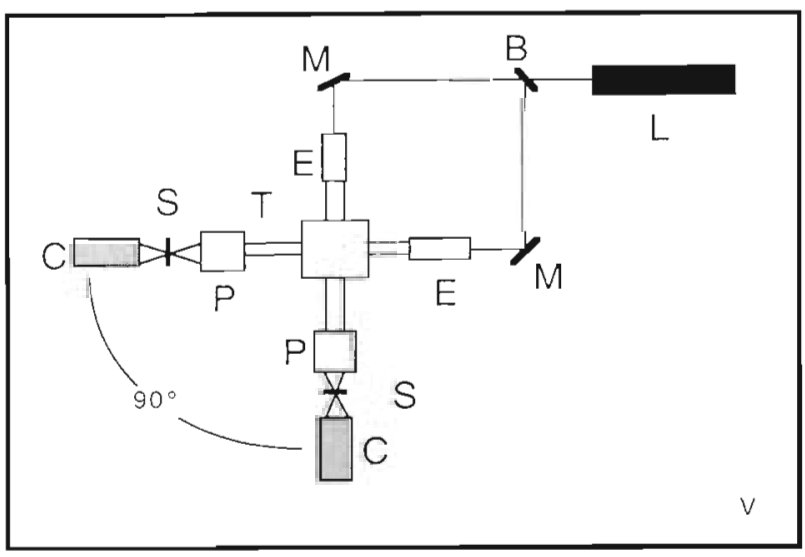

Fig. 1 Experimental apparatus as seen from above. B: beam splitter; C: CCD video camera; $E$ : beam expander; L: He-Ne laser; M: mirror; P. $35 \mathrm{~mm}$ camera lens; S: glass slide with Schlerian point; $\mathrm{T}$. glass tank; $\mathrm{V}$ : vibration isolation table ously. For the first minute, the copepods were illuminated by a helium/neon laser (632 nm) only, which produced a red light that did not appear to affect their behavior. After 1 min, a fiber optic light cable projecting down into the tank was turned on. This light was left on for 7 min and then turned off. The copepods were then recorded for 2 min more with only the red laser for illumination. After each experiment, the copepods were preserved in formalin solution so they could be counted and their antennae and prosome lengths measured.

For all species except Coullana canadensis, the end of the fiber light cable was capped with a white plastic test-tube stopper to diffuse the light into a wider conical shape (Fig. 2). The diffuser also made the width of downward emitted light fill most of the horizontal width of the video's field of view, and provided a physical barrier to keep copepods from escaping above the light and out of the view of the cameras. Due to their much smaller size and the higher level of video magnification, the diffuser was unnecessary for the $C$. canadensis nauplii.

For 4 species, 5 different short $(<3$ s) time sequences from the period when the fiber optic light was on were analyzed from within the same 10 min experiment, to

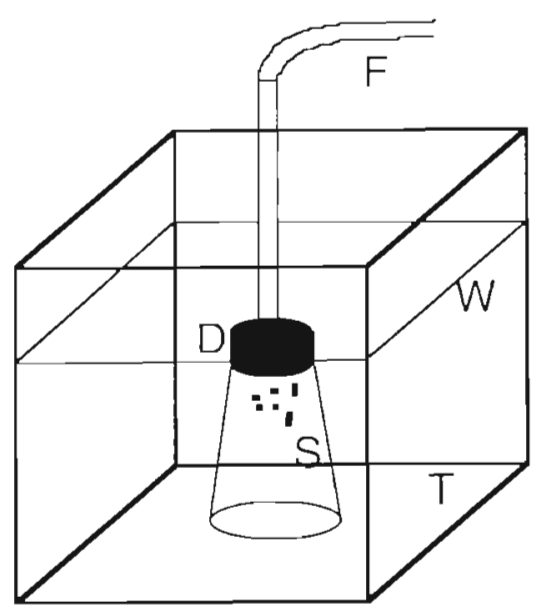

Fig. 2. Tank and diffuser apparatus. D: light diffusion apparatus; F: fiber optic cable; T. glass tank; W water level 


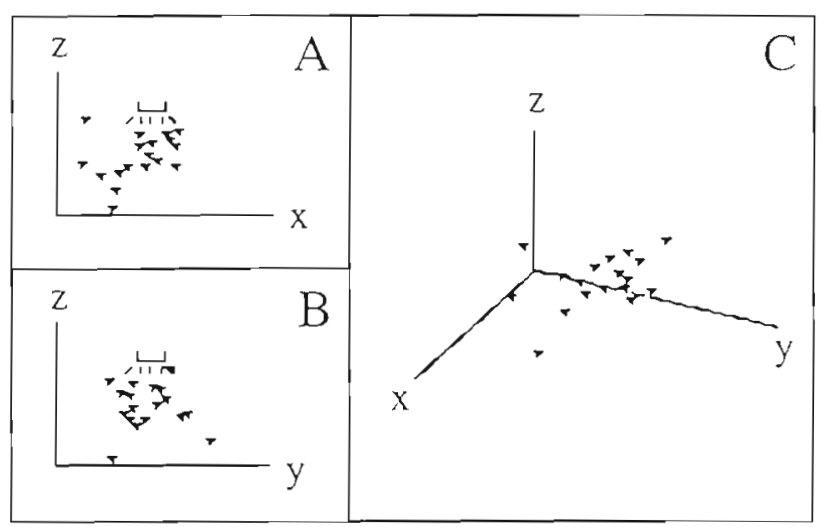

Fig. 3. Coullana canadensis. The matching problem. (A) Twodimensional view of a swarm in the $(x, z)$ plane. (B) Twodimensional view of a swarm in the $\{y, z)$ plane. C) Representation of the true 3 -dimensional swarm over the $(x, y, z)$ volume

obtain 3D position information. However, only 3 short sequences were analyzed for Diaptomus sp., 1 containing no mating attempts, the second containing a single mating attempt, and the third containing 2 different mating attempts. For each of the 5 short time sequences, positions of Coullana canadensis were recorded once every 4 frames for a total of 16 time points per individual, equaling a total time interval of $2.14 \mathrm{~s}$ (64 frames) per sequence. The length of time and interval between datum acquisition were chosen based on the minimum number of data points with different enough positions to conduct the matching of individuals. For all other species, which moved faster and more often than the C. canadensis nauplii, positions were recorded once every 2 frames for a total of 10 time points, equaling a $0.666 \mathrm{~s}$ interval (20 frames) for each of the 5 short sequences ( 3 for Diaptomus sp.).

To calculate the nearest-neighbor distance (NND), it is necessary to determine the $3 \mathrm{D}(x, y, z)$ coordinates of all members of a swarm at a specific time. This is no easy task due to the 'matching' problem discussed by Ikawa et al. (1994). The matching problem arises when trying to match images of individuals from orthogonal views of the same swarm when there are no characteristics to distinguish individuals (Fig. 3) To solve this problem the following novel method was developed. Multiple 2-dimensional (2D) $(x, z)$ and $(y, z)$ tracings were made of the positions of individuals in each swarm on acetates laid over a video monitor. Marks on the acetates were made as a dot as near as possible to the middle of the body. The $2 \mathrm{D}$ acetate tracings were then digitized. Acetate alignment was accomplished by the marking of 2 vertical alignment dots on each acetate corresponding with dots on the video monitor and on the digitizing tablet. Once the 2 corresponding acetates ( 1 from each angle) were digitized, the two 2D

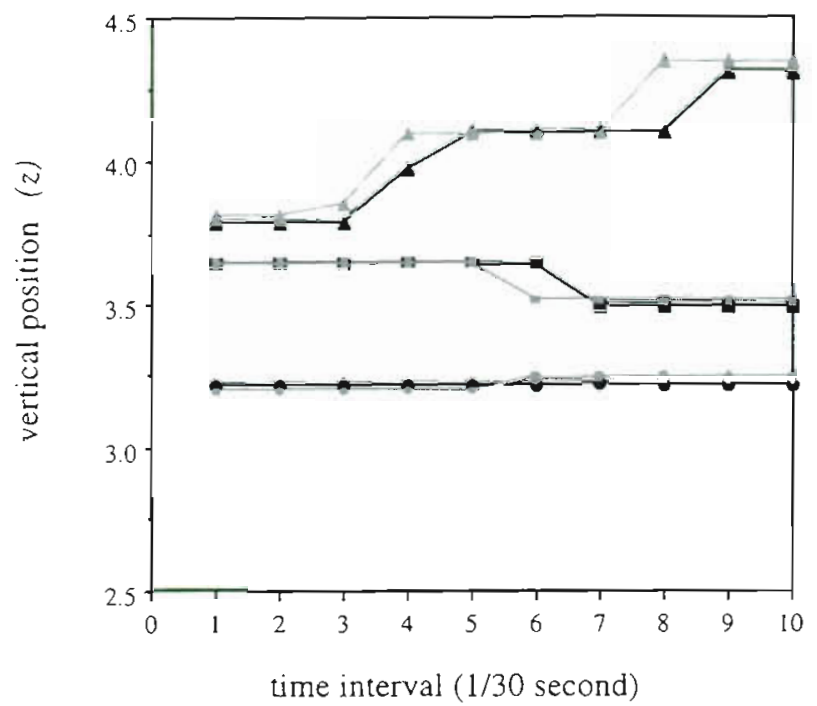

Fig. 4. Coullana canadensis. Vertical positions over time of 3 copepods from 2 different angles. Grey symbols and lines represent individuals from view $A$, while black symbols and lines represent the corresponding individuals from view $B$

data files were matched using a computer program (written in Basic and run on a Mac II). The matching algorithm was based on the premise that a single individual would have the same absolute vertical position and the same relative change in vertical position $(\mathrm{d} z / \mathrm{d} t)$ over time from either $2 \mathrm{D}$ view (Fig. 4). Both absolute vertical position and change in vertical position were unique enough for the individual's 2 tracks to be matched. The lag in vertical position changes between the 2 tracks of an individual were due to slight differences in the digital timer coding for each video, but time points were at most $1 / 30$ s off between the 2 videos and did not affect the overall matching ability of the program.

One data file was produced, containing the 3D coordinates of all individuals over time, and their corresponding nearest-neighbor values, from which the average NND for each time point within each time sequence could be calculated. These averages were then summed and divided by the number of frames per sequence in order to calculate the overall average NND for each short time sequence. Also, the mean minimum nearest-neighbor distance (MNND) was calculated as the sum of the shortest distance between 2 copepods out of all possible pairings in the swarm for each particular frame, divided by the number of frames analyzed (10, or 16 for Coullana canadensis) It also was possible to directly calculate the velocity of each individual copepod from each swarm by dividing the distance between each subsequent spatial position by the time interval between sequential positions. 
The density for each short time sequence was calculated by finding the spherical volume occupied by the swarm and dividing this by the number of copepods in the swarm. The diameter defining the spherical volume was determined by taking the average of the furthest separated copepods vertically and the furthest separated copepods horizontally. This method seemed to include the least amount of water containing no swarming individuals.

The measured MNND described above was then compared with the predicted MNND calculated by an equation from Rothschild (1992):

$$
\text { MNND }=0.55 n^{-1 / 3} \text { density }{ }^{-1 / 3}
$$

where $n$ is the number of copepods in the swarm. This equation assumes that the copepods are distributed randomly (Poisson distribution) in space and that the MNND should decrease as the density of the swarm increases. The measured MNND versus density was in turn compared to each of the predicted calculations of MNND versus density. Comparisons were made using $t$-tests for each of the 5 different swarm densities for each species, and then setting the alpha level to 0.05 for the sum of the 5 separate $t$-tests (alpha $=0.01$ for each individual $t$-test). Goodness-of-fit tests between the measured and predicted distributions were not used, as they do not have as much power as a $t$-test when the sample variance is known.

To test the computer program's accuracy, $1 \mathrm{com}$ puter-matched sequence was compared to human matching of the same sequence. The human matching was accomplished by viewing graphs of the vertical positions over time of all individuals from one view versus all individuals from the other view. The computer program was found to have mismatched 2 pairs out of 21 total pairs. However, the program provides a goodness-of-matching (GOM) index for each match, such that the user can easily see if the matching index is poor In the case of the test sequence, the GOM index number was poor for the mismatched pairs. In subsequent sequences, poorly matched pall as indicated by the GOM index, were eliminated from the calculations. This was a valid procedure, as mismatched individuals were the result of an individual appearing in one view and not the other, since the depth of field from a camera was not exactly the same as the width of field from the other camera.

\section{RESULTS}

We wished to determine if swarm members stay a specific distance apart from each other and if this distance is defined by the actual exoskeletal boundaries or by an additional layer of water modified by the pres- ence of the moving zooplankter. In this experiment we were interested in the absolute minimum nearestneighbor distance and its relationship to the size of the zooplankter, here measured as the size of the antenna or prosome. To test this, we compared the measured MNND versus a predictor of MNND based on the copepods being randomly distributed, which also predicts that as density increases or number of swarming individuals increases the MNND should decrease. If the copepods were keeping further away from each other than predicted (measured MNND > predicted MNND) then they were not randomly distributed, suggesting that they were actively maintaining a space around themselves within which they would not allow others to come.

The measured MNND for Acartia tonsa was significantly greater than the predicted MNND for all 5 swarm densities (Fig. 5) ( $p<0.01$ ). For Oithona sp., the MNND was significantly greater than the predicted MNND for 4 out of the 5 different swarm densities (Fig 5) $(p<0.01)$. At the highest swarm density, the measured MNND was less than but not significantly different from the predicted MNND ( $p>0.01$ ). For the harpacticoid and Coullana canadensis, the measured MNND was not significantly different from the predicted MNND.

Diaptomus sp. appeared to be attempting to mate in the second and third trials that we analyzed. In the videos it could be seen that one copepod was clasping onto another using its geniculate antenna. The MNND for the first trial showed the normal avoidance minimum, while the MNND values for the second and third trials were much lower than the normal avoidance minimum because of the mating attempts. The MNNDs of $<0.45 \mathrm{~mm}$ (Fig. 5) are much smaller than the single antenna or the prosome length (Table 2), showing that Diaptomus sp. must have been coming into physical contact at times. Therefore, MNNDs measured during mating were not used in the comparison of measured MNND with predicted MNND. For

Table 2. Prosome length and single antenna length (in $\mathrm{mm}$, mean \pm standard deviation, number of replicates given in parentheses) for the 5 studied species

\begin{tabular}{|lcc|}
\hline Species & Prosome length & Antenna length \\
\hline Acartia tonsa & $0.61 \pm 0.084(20)$ & $0.65 \pm 0.097(20)$ \\
Harpacticoid & $0.31 \pm 0.012(20)$ & $0.30 \pm 0.014(20)$ \\
Diaptomus sp. & $0.92 \pm 0.055(20)$ & $0.86 \pm 0.067(20)$ \\
Oithona sp. & $0.32 \pm 0.043(20)$ & $0.33 \pm 0.046(20)$ \\
Coullana canadensis & $0.20 \pm 0.026(82)$ & $0.20^{\circ}$ \\
anda were not available on the antenna size for C. \\
Canadensis in this experiment, so an estimate of $0.2 \mathrm{~mm}$ \\
was used
\end{tabular}



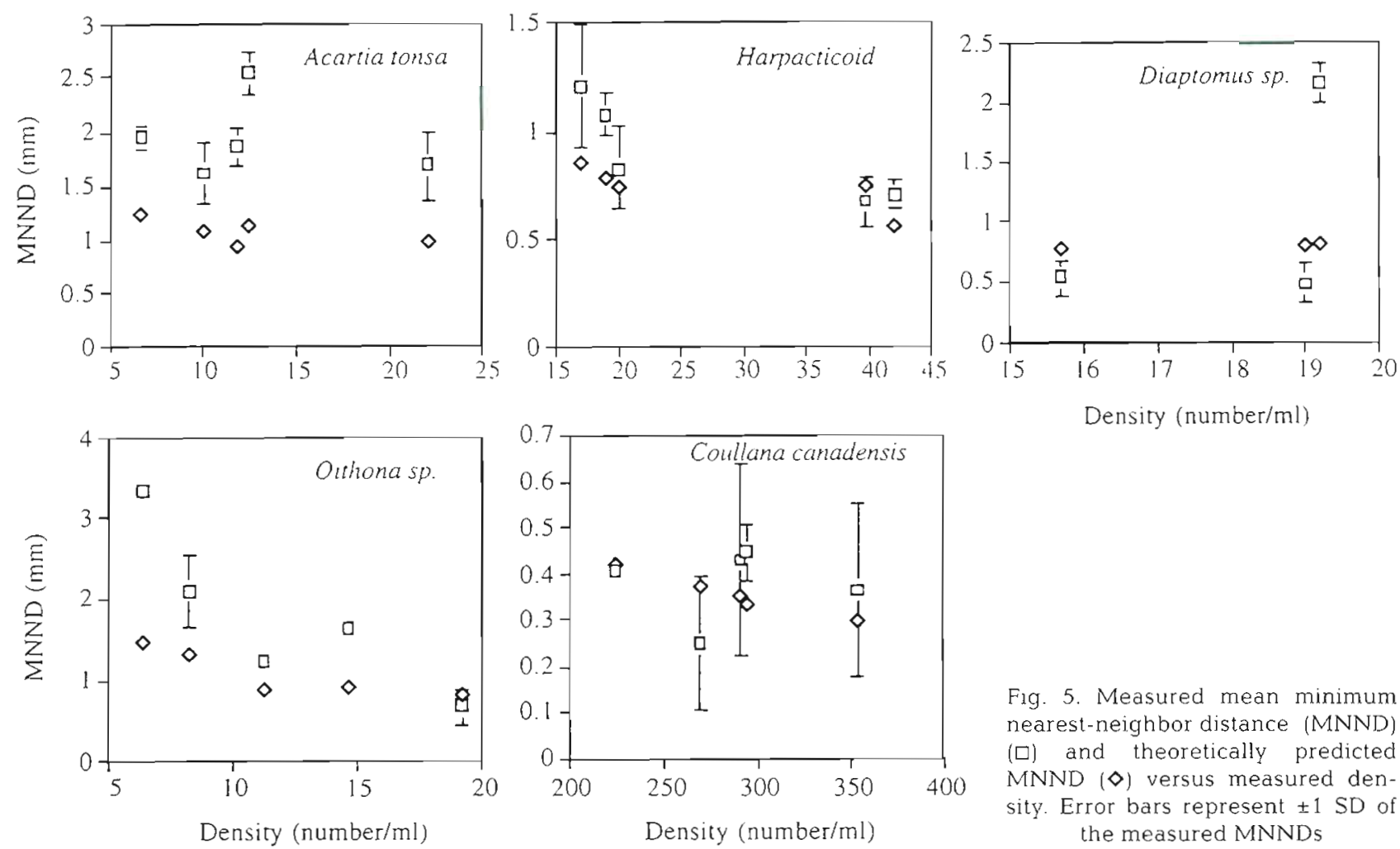

Fig. 5. Measured mean minimum nearest-neighbor distance (MNND) $(\square)$ and theoretically predicted MNND $(\diamond)$ versus measured density. Error bars represent \pm 1 SD of the measured MNNDs

the non-mating sequence, the measured MNND was significantly greater than the predicted MNND $(\mathrm{p}<$ $0.01)$.

Average prosome length and average antenna length (Table 2) were both found to be significantly correlated with the average MNND for the 3 species which had MNNDs greater than the predicted MNND

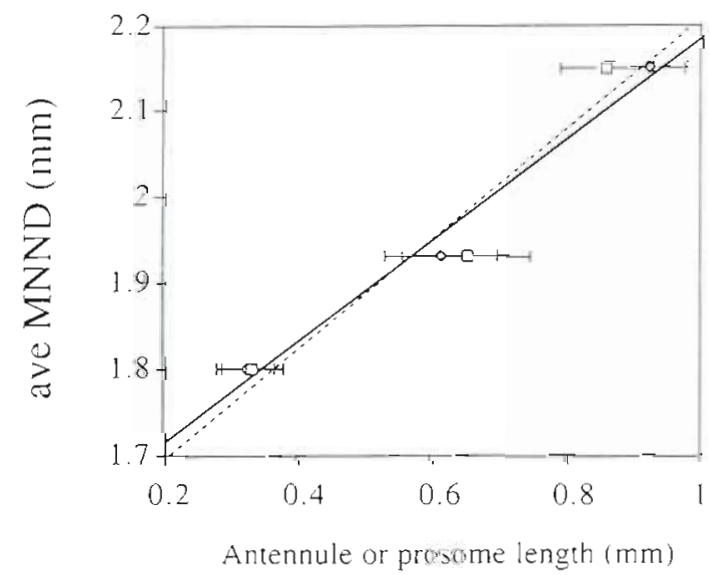

Fig. 6. Measured MNND versus antennule length $(\square$ and $\cdots$ ), and prosome length ( $\diamond$ and -), for Oithona sp., Acartia tonsa, and Diaptomus sp. Equation for line is $y=0.64 x+1.57$ $(r=0.965)$ for MNND versus antennule, and $y=0.59 x+1.6$ $(r=0.992)$ for MNND versus prosome
(Fig. 6). For all 3 species, the MNND was greater than twice the antennule or prosome length, such that physical contact between individuals' exoskeletons did not occur.

Swarm densities ranged from several times to orders of magnitude higher than the average density within the test tank (Table 1). The highest maximum swarm density of the 5 species was 353 copepods $\mathrm{ml}^{-1}$ for Coullana canadensis nauplii, while the lowest maximum swarm density was 19.2 copepods $\mathrm{ml}^{-1}$ for Diaptomus sp. and Oithona sp. (Table 1)

Video observations revealed that the 5 species exhibited different swimming speeds and behaviors which affected the morphology of the swarm. We therefore calculated the average sinking speed, average cruising speed, and maximum jump speed for each species using the 3D positional data obtained from the matching program (Table 3). Acartia tonsa exhibited the highest jump speed of $87 \mathrm{~mm} \mathrm{~s}^{-1}$, while the Coullana canadensis nauplii were the slowest at $13 \mathrm{~mm} \mathrm{~s}^{-1}$ (Table 3). The other 3 species had similar maximum jump speeds ranging from 35 to $39 \mathrm{~mm} \mathrm{~s}^{-1}$ (Table 3 ). Sinking rate ranged from 1 to $0.1 \mathrm{~mm} \mathrm{~s}^{-1}$, except for Diaptomus sp. which did not appear to sink at all. Average swimming speed ranged from $2.9 \mathrm{~mm} \mathrm{~s}^{-1}$ for C. canadensis nauplii to $5.6 \mathrm{~mm} \mathrm{~s}^{-1}$ for $A$. tonsa, but in all cases except the adult harpacticoid, the standard deviation was greater than the average value. 
Table 3. Swimming speed, sinking speed (in mm s${ }^{-1}$, mean \pm standard deviation, number of replicates given in parentheses), and the maximum speed seen for all individuals (in $\mathrm{mm} \mathrm{s}^{-1}$ )

\begin{tabular}{|lcccc|}
\hline Species & $\begin{array}{c}\text { Swimming } \\
\text { speed }\end{array}$ & $\begin{array}{c}\text { Sinking } \\
\text { speed }\end{array}$ & $\begin{array}{c}\text { Max } \\
\text { speed }\end{array}$ \\
\hline Acartia tonsa & $5.6 \pm 10.9(268)$ & $0.85 \pm 0.26(6)$ & 87 \\
Harpacticoid & $4.0 \pm 3.6$ & $(386)$ & $0.52 \pm 0.09(6)$ & 36 \\
Diaptomus sp. & $3.6 \pm 3.8(431)$ & 0. & 39 \\
Oithona sp. & $3.7 \pm 4.4$ & $(112)$ & $0.24 \pm 0.05(6)$ & 35 \\
Coullana & $2.9 \pm 4.2$ & $(163)$ & $0.17 \pm 0.04(6)$ & 13 \\
canadensis & & & \\
aiaptomus sp. appeared to be actively swimming down- \\
wards and not to be sinking \\
\hline
\end{tabular}

The combined effect of different swimming speeds and behavior appeared qualitatively to affect the flux of individuals into and out of a swarm. Acartia tonsa, a pelagic calanoid and the second-largest of the species studied, cruised slowly by beating its mouth parts. During encounters or excitation, A. tonsa exhibited jump behavior, using its tail and antenna. A. tonsa rarely stopped moving while swarming, as their sinking rate was great enough that within a few seconds they were out of the highest lit areas, reactivating the phototactic response to swim back to the light (Table 3). These characteristics suggest that $A$. tonsa swarms have a high flux rate of individuals. Oithona sp., a pelagic cyclopoid copepod, exhibited only fast and slow cruise speeds (Table 3) and appeared relatively inactive during swarming. Their very slow sinking speed enabled them to remain within the upper, highest lit areas for a much longer period, suggesting that Oithona sp. swarms have a low flux rate of individuals. The benthic harpacticoid that was studied exhibited cruise and jump behavior, although there was less difference between the velocities of the 2 modes than for A. tonsa (Table 3). While the other species had spherical swarms, the harpacticoid swarm was drawn out vertically due to its high sinking speed. The late naupliar stage of Coullana canadensis exhibited 2 swimming modes: directional swimming and circular swimming. On a distant approach to the light source, $C$. canadensis swam in relatively straight lines, while within the high-light areas, they exhibited circular swimming which allowed them to keep moving yet remain under the light shaft. Due to their small size and swimming pattern, encounters should have been minimized and flux rate should have been low. Diaptomus sp., a freshwater calanoid and the largest of the species studied, exhibited cruise and jump behavior, with much less difference in speed between the 2 behaviors than in $A$. tonsa (Table 3). Diaptomus sp. individuals were very active in the swarm, and never seemed to be at rest. They appeared never to sink away from the light, but to cruise down and away from the light, only to turn around and cruise back towards the light upon reaching a low-light area. Their high activity levels suggest that Diaptomus sp. swarms also have high flux rates of individuals into and out of the swarm

\section{DISCUSSION}

Physical contact is the absolute minimum distance between copepods. However, our quantitative visual analysis of closest packing of 5 species of swarming copepods showed that swarm members of at least 3 species remained separated and did not come into body-to-body contact (except for mating in 1 case). As zooplankters can move to alter their spacing, we considered the mechanisms maintaining their separation. One is behavioral (active), where perception of a neighbor elicits an escape response, resulting in separation of individuals and flux of copepods out of the swarm. Another is physical (passive), where a layer of water-surrounding the copepod as the boundary layer of its feeding current-separates individuals. Since there are streamlines generated by a copepod's feeding current which do not intersect with any portion of their exoskeleton (Fields \& Yen 1993), individuals could be drawn towards each other and then advected past one another without coming into physical contact for a certain number of relative orientations.

We found that the minimum NND was greater than twice the antennule length. Fields \& Yen (1993) found that the volume of water significantly affected by the feeding current of a large omnivorous copepod Pleuromamma xiphias was approximately 175 times the actual volume of the copepod, and hypothesized that the sensory abilities of a copepod should extend just beyond this volume of self-generated disturbance in order to properly perceive prey items. If this holds true for the copepods in these experiments, then at a separation distance of the measured MNND, the feeding currents of these copepods would have a fairly large overlap region, indicating that they may sense, yet ignore, each other before the minimum separation distance is reached. Conversely, it may be that these copepods do not sense beyond the range of the strongest parts of their feeding currents, making the MNND a direct measure of their perceptive range for similar sized organisms to themselves.

If copepods within a swarm sense each others' feeding currents at distances greater than the MNND, the mechanism which maintains separation must involve an active component, whereby individuals suppress their normal escape response when they first sense a 


\begin{tabular}{|c|c|c|c|c|}
\hline Time & Copepc & position & View 1 & View 2 \\
\hline & No. 1 & No. 2 & & \\
\hline 1 & a & d & & \\
\hline 2 & a & $d$ & Copepod 1 & Copepod 1 \\
\hline 3 & a & $e$ & & \\
\hline 4 & a & e & & \\
\hline 5 & b & e & & 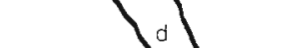 \\
\hline 6 & $b$ & e & & Cocerid 2 \\
\hline $\begin{array}{l}7 \\
8\end{array}$ & $\begin{array}{l}b \\
b\end{array}$ & $f$ & & Cond \\
\hline 9 & C & g & & $\mathrm{L}$ \\
\hline 10 & c & g & & $1 \mathrm{rm}$ \\
\hline
\end{tabular}

Fig. 7. Acartia tonsa. Example of an escape response. Positions of 2 individual copepods in a swarm during 1 time sequence of 10 time points. The 2 views represent the same individuals seen from the 2 orthogonal camera positions. Copepod 1 starts at position a at time 1. Copepod 2 starts at position $d$ at time 1. At time 8 copepod 1 is at position $b$, and copepod 2 is at position $\mathrm{f}$. Between time 8 and 9 \{1/15 s\}, copepod 1 travels from position $b$ to position $c$, a path which takes it within the detection range of copepod 2, eliciting an escape response by copepod 2

nearby neighbor. As individuals get physically closer, either an escape response or the interaction of the nonbody intersecting streamlines of the 2 individuals' feeding currents prevents closer contact. It is not clear which of the 2 mechanisms is more important in maintaining the MNND, as it was not possible to match all the occurrences of escape responses with specific NND measurements. However, we were able to match a few escape responses to times where 2 individuals came within the MNND of each other (Fig. 7), showing that the fully active mechanism occurs. Also, because the Diaptomus sp. upon close encounters had either active escapes or mating attempts (body to body contact occurred), at least this species can sense near neighbors at distances beyond its physical body and at times ignores this information and does not attempt an escape

There are several possible explanations for the 2 species whose distributions did not deviate from the random spacing model. The first is that the densities of the swarms were not high enough to cause a departure from the random spacing model. This would imply that below some critical density, individuals within a swarm are randomly distributed, but above that density, interactions between swarming individuals cause a departure from a random distribution, with greater than expected MNNDs. The second possibility is that these 2 copepod groups do not collect the same type of mechanosensory information as the other 3 types. Harpacticoids have a relatively small ratio of first antenna length to body size compared to the other groups, a fairly different antennule morphology from the calanoids, and usually quite different lifestyles. It may be that they do not rely on mechanoreception, bul more on chemoreception. The nauplii studied do not have fully developed antennules like the adults that were used, suggesting that they may also be limited in their mechanosensory abilities.

The results obtained in these experiments have several implications for naturally occurring assemblages of copepods. The first is that for some species, the distribution of individuals within a swarm may not be randon' at high densities, as we were able to reject the random model based on our MNND measurements. The cause of this departure from a random distribution is most likely due to the spacing mechanisms discussed above, causing a truncation of inter-individual distances smaller than the MNND and leading to a more even distribution of copepods throughout a swarm. If swarming individuals in situ are not randomly distributed, then it is not appropriate to estimate densities from measurements of average inter-individual distances (or vice versa) using prediction models based on random spacing. The directly measured swarm densities in our experiments, however, are much greater than any measured in the field (Acartia australis, $0.5 \mathrm{ml}^{-1}$. Oithona oculata, $1.5 \mathrm{ml}^{-1}$, Hamner \& Carlton 1979; Acartia plumosa, $2 \mathrm{ml}^{-1}$, O. oculata, $0.4 \mathrm{ml}^{-1}$, Udea et al. 1983; Diothona oculata, $23 \mathrm{ml}^{-1}$, Ambler et al. 1991) Field measurements of average NND, MNND, and density are needed to determine whether these lowerdensity, in situ swarmers are also not randomly distributed.

The second implication of this study is that copepods may selectively ignore mechanosensory information. In one of the spacing mechanism scenarios, we hypothesized a suppression of the normal escape response, because the MNND was smaller than the likely ambit of the feeding current. If suppression of reaction is occurring, we must consider the factors which could be causing this suppression. One is that chemosensory cues may be present, such as pheromones or certain amino acids denoting the presence of conspecifics (Poulet \& Ouellet 1982), which could suppress the escape response. Another is that copepods could possibly recognize the 'signature' of a conspecific-its species and stage specific feeding current or swimming movements (Strickler 1985) - and therefore not need to escape. This possibility could be tested by placing a similarly sized individual of a different species into a swarm and noting any changes in MNNDs. The third possibility is that normal escape responses were suppressed by the light that was used to induce the swarm, and exhibiting positive phototaxis was more 
important to these copepods than responding to encroaching neighbors.

Reductions in swarm density with distance from the swarm marker, the light (Yen \& Bryant 1992), combined with our behavioral observations from these experiments suggest that this type of swarm can be divided into 3 zones. In zone 1, closest to the light, the high density of the closely packed copepods causes escapes as well as sinking away from the light. In zone 2 , farther away from the light, the encounter rate is lower than in zone 1 ; there are fewer escapes; the main copepod movements involve sinking away from the light and swimming back toward it. In zone 3 , the furthest areas from the light, there are more turns back into the swarm and oriented swimming toward the light source. Our qualitative observations suggest that speed and heading angle vary within these zones and should therefore affect swarm morphology (Leising \& Yen unpubl.). Detailed observations of velocity versus distance from the stimulus may also reveal further information about the mechanisms used to maintain spacing within swarms and the nature of the suppression reaction. Future research could also be aimed at examining swarm formation in response to other cues such as odors or at assessing how much turbulence is needed to disperse a swarm.

Acknowledgements. This research was supported by the National Science Foundation OCE-9314134 and the Office of Naval Research N-00014-94-10696 to J.Y Work on the manuscript was partially supported by NOAA NA36GP0320, and ONR N00014-95-1-0189 and N00014-95-1-0764 to Peter J. S. Franks. A.L. thanks Elof A. Carlson and the Honors College at Stony Brook for initiation of this project. A.L. also especially thanks P. J. S. Franks for help in revising the manuscript and the 2 anonymous reviewers for their excellent suggestions and critrques of further revisions. This is Contribution No. 1062 from the Marine Sciences Research Center at the State University of New York at Stony Brook.

\section{LITERATURE CITED}

Ambler JW, Ferran FD, Fornshell JA (1991) Population structure and swarm formation of the cyclopoid copepod Diolthona oculata near mangrove cays. J Plankton Res 13: $1257-1272$

Clutter RI (1969) The microdistribution and social behavior of some pelagic mysid shrumps. J Exp Mar Biol Ecol 3:125-155

Davis CS, Flierl GR, Wiebe PH, Franks PJS (1991) Micropatchiness, turbulence, and recruitment in plankton. J Mar Res 49:109-151

Fields D, Yen J (1993) Outer limits and inner structure - the 3-dimensional flow field of Pleuromamma xiphias (Calanolda, Metridinidae). Bull Mar Scl 53:84-95

Gill CW (1985) The response of a restrained copepod to tactile stimulation. Mar Ecol Prog Ser 21:121-125

Gill CW (1986) Suspected mechano- and chemosensory structures of Temora longicornis (Copepoda: Calanoida). Mar Biol 93:449-457

Hamner WH, Carlton JH (1979) Copepod swarms: attributes and role in coral reef ecosystems. Limnol Oceanog 24:1-14

Haury LR, Kenyon DR, Brooks JR (1980) Experimental evaluation of the avoidance reaction of Calanus tinmarchicus. J Plankton Res 2.187-202

Haury LR, Wiebe PH (1982) Fine-scale multi-species aggregatıons of oceanic zooplankton. Deep Sea Res 29:915-921

Hebert PDN (1988) Induced swarming in the predatory copepod Heterocope septentrionalis. Limnol Oceanogr 25: $747-750$

Ikawa T, Okabe H, Mori T, Urabe K, Ikesoji T (1994) A method for reconstructung 3-dimensional positions of swarming mosquitoes. J Insect Behav 7:237-248

Legier-Visser MF, Mitchell JG, Okubo A. Fuhrman JA (1986) Mcchanoreception in calanoid copepods, a mechanısm for prey detection. Mar Biol 90:529-535

Lenz PH, Yen J (1993) Distal setal mechanoreceptors of the first antenna of marine copepods. Bull Mar Sci 53:170-179

Omori M, Hamner WH (1982) Patchy distribution of zooplankton: behavior, population assessment, and sampling problems. Mar Biol 72:193-200

Paffenhöfer GA, Lewis KD (1990) Perceptive performance and feeding behavior of calanoid copepods. J Plankton Res 12:933-946

Paffenhöfer GA, Strickler JR, Alcaraz M (1982) Suspensionfeeding by herbivorous calanoid copepods: a cinematographic study. Mar Biol 67:193-199

Partridge $B$, Pitcher $T$ (1980) The sensory basis of fish schools: relative roles of lateral line and vision. J Comp Physiol 135:315-325

Poulet SA, Ouellet G (1982) The role of amino acids in the chemosensory swarming and feeding of marine copepods. J Plankton Res 4:341-361

Rotschild BJ (1992) Applications of stochastic geometry to problems in plankton ecology. Phil Trans R Soc Lond Ser B $336: 225-237$

Strickler JR (1975) Intra- and interspecific information flow among planktonic copepods: receptors. Verh Int Verein Limnol 19:2938-2951

Strickler JR (1982) Calanoid copepods, feeding currents, and the role of gravity. Science 218:158-160

Strickler JR (1985) Feeding currents in calanoid copepods: two new hypotheses. In: Laverack MS (ed) Physiological adaptations of marine animals. Soc Exp Biol Semin Ser 39: $459-485$

Strickler JR, Bal AK (1973) Setae of the first antennae of the copepod Cyclops scutifer (Sars): their structure and importance. Proc Nat Acad Sci USA 70:2656-2659

Udea H, Kuwahara A, Tanaka M. Azeta M (1983) Underwater observations on copepod swarms in temperate and subtropical waters. Mar Ecol Prog Ser 11:165-171

Wiebe PH (1970) Small-scale spatial distribution in oceanic zooplankton. Limnol Oceanogr 15:205-217

Yen J (1985) Selectuve predation by the carnivorous marine copepod Euchaeta elongata: laboratory measurements of predation rates verified by field observations of temporal/ spatial feeding patterns. Limnol Oceanogr 30:577-595

Yen J, Bundock EA (in press) Aggregate behavior in zooplankton: phototactic swarming in 4 developmental stages of Coullana canadensis (Copepoda, Harpacticoida). In: Parish J, Hamner J (eds) Clumps: from individuals to aggregations. Cambridge University Press, Cambridge

Yen J, Lenz PH, Gassie DV, Hartline DK (1992) Mechanoreception in marine copepods-electrophysiological studies on the first antenna. J Plankton Res 14:495-512

Yen J, Sanderson B, Strickler JR, Okubo A (1991) Feeding currents and energy dissipation by Euchaeta rimana, a subtropical pelagic copepod. Limnol Oceanogr 36:362-369 